\title{
Klimawandel und Sicherheit im Nahen Osten
}

\author{
Hans Günter Brauch*
}

\begin{abstract}
The year 2007 was a turning point for a securitization of climate change for international (UK, Germany, EU), national (USA) and human (Human Security Network, GECHS) security. The complex linkages between the earth and human systems are illustrated with the PEISOR model. One of the most affected regions with a limited problem awareness on climate change is the Near East. The effects, impacts and societal outcomes of climate change for security require a policy response by the countries in the Near East and by the European Union to avoid that these trends may pose security dangers. A solar initiative in the framework of the Mediterranean Union is suggested as part of a transformation strategy.
\end{abstract}

Keywords: Klimawandel, Sicherheit, Naher Osten, Konfliktkonstellationen, Solarinitiative

\section{Einleitung und Fragestellung}

$\mathrm{K}$ limawandel wird seit Anfang des 21. Jahrhunderts als Problem der internationalen, europäischen, nationalen und menschlichen Sicherheit interpretiert. ${ }^{1}$ Folgende Ereignisse haben dazu beigetragen, dass das Jahr 2007 als Wendepunkt für die „Versicherheitlichung“2 ${ }^{\text {des anthropogen }}$ verursachten globalen Klimawandels angesehen wird:

- 2007 legte der Intergovernmental Panel on Climate Change (IPCC) seinen vierten Bericht vor, womit der Klimawandel hohe politische Aufmerksamkeit erzielte.

- Im April 2007 setzte Großbritannien den Klimawandel auf die Tagesordnung des VN-Sicherheitsrats.

- Im Juni 2007 legte der Wissenschaftliche Beirat der Bundesregierung Globale Umweltveränderungen (WBGU) sein Jahresgutachten: Sicherheitsrisiko Klimawandel vor und Ende Juni 2007 bat die Bundesregierung die Europäische Union um eine Stellungnahme zu Klimawandel und internationaler Sicherheit, die am 14. März 2008 vorgelegt wurde.

- Am 12. Oktober 2007 erhielten der IPCC und der ehemalige US-Vizepräsident Al Gore den Friedensnobelpreis für ihr Engagement bei der Bekämpfung des Klimawandels.

- Am 12. Dezember 2008 nahm der Europäische Rat einen Bericht des Generalsekretärs/Hohen Vertreters „über die Umsetzung der Europäischen Sicherheitsstrategie“ an, worin der Klimawandel als „Bedrohungsmultiplikator“ genannt wurde. $^{3}$

In der internationalen politischen und wissenschaftlichen Debatte zu den sicherheitspolitischen Folgen des Klimawandels sind drei Sicherheitskonzepte von Bedeutung. Während

\footnotetext{
PD Dr. Hans Günter Brauch, Otto-Suhr-Institut für Politikwissenschaft, FU Berlin; Senior Fellow, Institute for Environment and Human Security, United Nations University, Bonn; Vorsitzender, AG Friedensforschung und Europäische Sicherheitspolitik (AFES-PRESS e.V.); Herausgeber der Hexagon-Buchreihe, Human and Environmental Security and Peace im Springer Verlag.

1 Vgl. H.G. Brauch, „Klimawandel, Umweltstress und Konflikt“, in: BMU (Hrsg.): Klimawandel und Konflikte (Berlin: BMU, 2002): 11-110; WBGU, 2007: Welt im Wandel - Sicherheitsrisiko Klimawandel (Berlin - Heidelberg: Springer).

2 Vgl. Wæver's Theorie der „Securization“, das Kernstück der Kopenhagener Schule zur Sicherheitsforschung. O. Wæver: Concepts of Security (Copenhagen: University of Copenhagen, Institute of Political Science, 1997).

3 Vgl. Rat der Europäischen Union, „Bericht über die Umsetzung der Europäischen Sicherheitsstrategie - Sicherheit schaffen in einer Welt im Wandel“, Brüssel, 10.12.2008, Dokument 17104/08.
}

Konzepte der internationalen Sicherheit die britischen und deutschen Beiträge sowie die EU-Dokumente bestimmen, behandele die amerikanische Debatte Folgen für die nationale Sicherheit. Das Wissenschaftsnetzwerk GECHS und das Human Security Network (HSN) diskutieren Klimafolgen für die Menschen. ${ }^{4}$

Dabei ist der Klimawandel - neben Wasserfragen ${ }^{5}$ und Problemen der Desertifikation ${ }^{6}$ - nur einer von mehreren Problembereichen des internationalen Umweltwandels ${ }^{7}$, die seit dem Jahr 2000 als Sicherheitsfragen diskutiert werden.

Dieser Beitrag analysiert zunächst mithilfe des PEISOR-Modells Folgen des Klimawandels und davon bewirkte hydro-meteorologischen Naturkatastrophen, die sicherheitspolitische Bedrohungen, Herausforderungen, Verwundbarkeiten und Risiken verursachen, ${ }^{8}$ auslösen oder bestehende Konfliktkonstellationen verschärfen können. Im Anschluss daran werden für den Nahen Osten die vier Konfliktszenarien des WBGU Berichts zum Sicherheitsrisiko Klimawandel erörtert und eine politische Antwort diskutiert, um die Klimafolgen durch eine kooperative Transformationsstrategie einzudämmen. Der Beitrag endet mit Schlussfolgerungen zur europäischen Sicherheit.

4 Vgl. H.G. Brauch: "Securitizing Global Environmental Change", in: H.G. Brauch, Ú. Oswald Spring, J. Grin, C. Mesjasz, P. Kameri-Mbote, N. Chadha Behera, B. Chourou, H. Krummenacher (Hrsg.): Facing Global Environmental Change: Environmental, Human, Energy, Food, Health and Water Security Concepts. Hexagon Series vol. 4 (Berlin - Heidelberg - New York: Springer-Verlag, 2009): 65-104; J. Scheffran: "Security Risks of Climate Change: Vulnerabilities, Threats, Challenges and Strategies", in: H.G. Brauch, Ú. Oswald Spring, C. Mesjasz, J. Grin, P. Kameri-Mbote, B. Chourou, P. Dunay, J. Birkmann (Hrsg.): Coping with Global Environmental Change, Disasters and Security - Threats, Challenges, Vulnerabilities and Risks. Hexagon Series vol. 5 (Berlin - Heidelberg - New York: Springer, 2010); S. Bauer: "Stormy Weather: International Security in the Shadow of Climate Change", in: Brauch, Oswald Spring, Mesjasz et al. (2010).

5 Vgl. Ú. Oswald Spring; H.G. Brauch: "Securitizing Water”, in: Brauch, Oswald Spring, Grin, et al. (2009): 77-204.

6 Vgl. zum ‘soil security' Konzept: H.G. Brauch; Ú. Oswald Spring: Securitizing the Ground; Grounding Security. UNCCD Issue Paper No.2 (Bonn: UNCCD Secretariat, May 2009); H.G. Brauch: "Desertification - A New Security Challenge for the Mediterranean? Policy agenda for recognising and coping with fatal outcomes of global environmental change and potentially violent societal consequences“, in: W. Kepner, J.L. Rubio, D. Mouat,F. Pedrazzini, Fausto (Hrsg.): Desertification in the Mediterranean Region. A Security Issue (Dordrecht: Springer, 2006): 11-85.

7 Vgl. das Security Handbook for the Anthropocene: H.G. Brauch, Ú. Oswald Spring, C. Mesjasz, J. Grin, P. Dunay, N. Chadha Behera, B. Chourou, P. Kameri-Mbote, P.H. Liotta (Hrsg.): Globalization and Environmental Challenges: Reconceptualizing Security in the 21 st Century. Hexagon Series vol. 3 (Berlin - Heidelberg - New York: Springer-Verlag, 2008); sowie die Bände IV und V der HexagonBuchreihe.

8 Vgl. H.G. Brauch: Threats, Challenges, Vulnerabilities and Risks in Environmental Human Security, Source 1/2005 (Bonn: UNU-EHS, 2006). 
Abb. 1: PEISOR-Model. (C) Hans Günter Brauch, Ursula Oswald Spring: Securitizing the Ground; Grounding Security. UNCCD Issue Paper No.2 (Bonn: UNCCD, 2009).

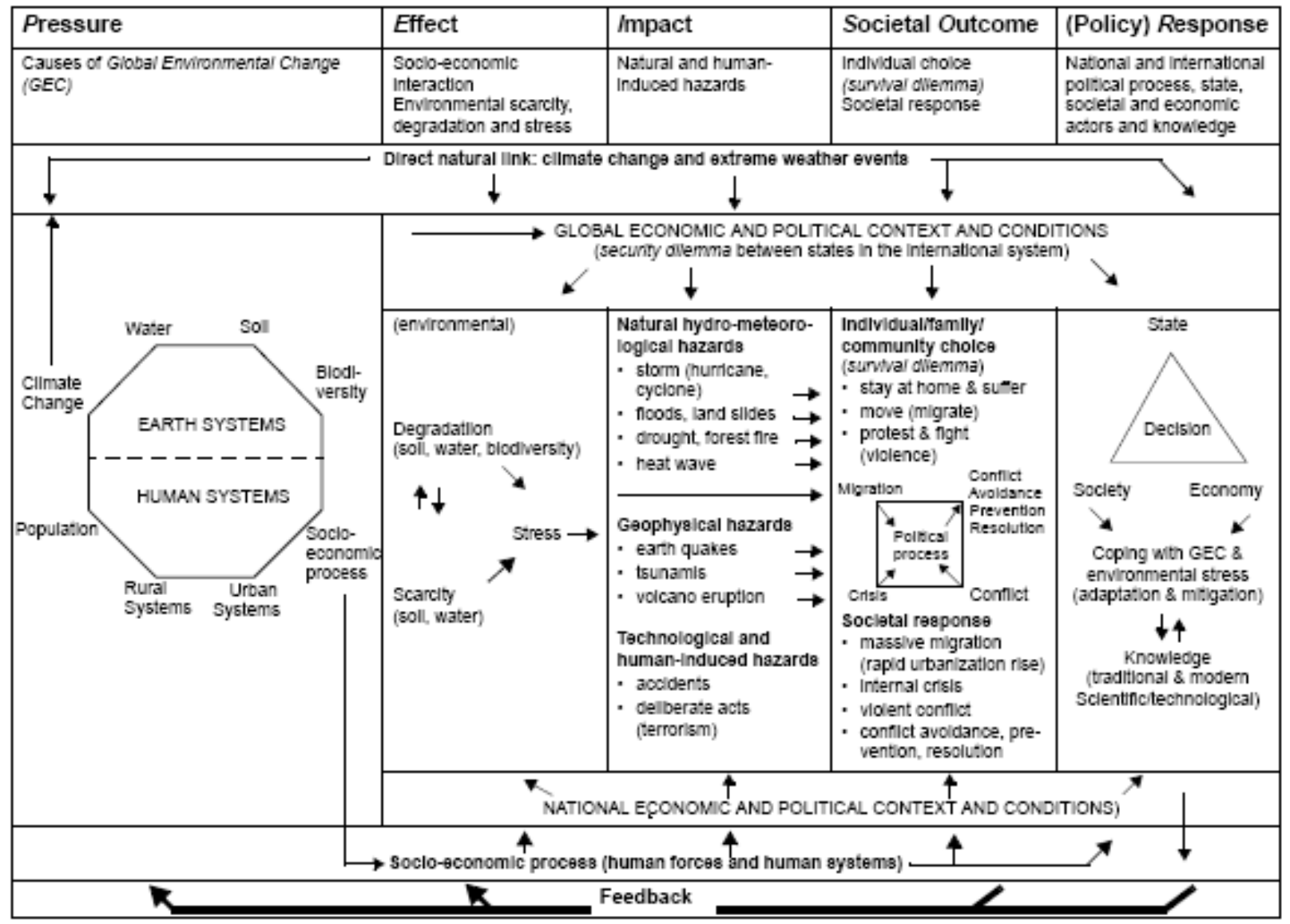

\section{Das PEISOR-Modell zur Analyse globaler Um- weltveränderungen}

Der globale Klimawandel verursacht einen Temperatur- und Meeresspiegelanstieg, Veränderungen der Niederschläge und eine Zunahme hydro-meteorologischer Naturkatastrophen. Die Interaktion von Faktoren des Erdsystems (Klimawandel, hydrologischer Zyklus, Bodendegradation, Biodiversität) mit dem menschlichen System erzeugt Druck auf das Erdsystem und auf sozio-ökonomische Prozesse, was zu diversen Sicherheitsproblemen führen kann.

Das PEISOR Modell unterscheidet zwischen fünf Phasen:

- P (pressure) bezieht sich auf acht Determinanten des globalen Umweltwandels;

- E behandelt die Wirkungen (effects) der linearen, nicht-linearen oder chaotischen Interaktionen innerhalb des Oktagons, die zur Verknappung von Ressourcen (Wasser, Böden), zu deren Degradierung durch Übernutzung und Umweltverschmutzung führen können, was häufig den Umweltstress erhöht;

- I (impacts) bezieht sich auf vom Menschen mit verursachte klimarelevante Naturkatastrophen (Dürre, Stürme, Überschwemmungen);
- SO (societal outcomes) behandelt sozio-politische Folgen von Umweltstress und Naturkatastrophen, insofern die Betroffenen mit suboptimalen Alternativen antworten, wie z.B. durch Binnenmigration, Landflucht und chaotische Urbanisierung, friedliche oder gewaltsame Massenproteste, was wiederum innenpolitische Krisen und Konflikte zur Folge haben kann.

- R (response) untersucht politische Antworten von Staat, Gesellschaft und Wirtschaft, wobei dem Wissen (Wissenschaft/ Technik) zur Ausarbeitung von Anpassungsstrategien große Bedeutung zukommt.

\section{Auswirkungen des Klimawandels im Nahen Osten}

Der Nahe Osten umfasst die außereuropäischen Länder am östlichen Mittelmeer, in diesem Beitrag die Türkei, Israel und fünf arabische Maschrek-Staaten: Syrien, Irak, Libanon, Jordanien und die besetzten Gebiete Palästinas (Abb. 2). Diese Region ist durch drei Konfliktmuster geprägt:

a) den Nahostkonflikt zwischen Israel und seinen arabischen Nachbarstaaten; 
Tabelle 1: Klimaverpflichtungen der Länder des Nahen Ostens.

\begin{tabular}{|c|c|c|c|c|c|c|c|c|c|c|c|c|}
\hline \multirow[t]{2}{*}{ Staaten } & \multicolumn{3}{|c|}{$\begin{array}{l}\text { UN-Klimarahmen-konvention } \\
\text { (UNFCCC) }\end{array}$} & \multicolumn{3}{|c|}{ Kyoto-Protokoll } & \multicolumn{4}{|c|}{$\begin{array}{c}\text { Nationale } \\
\text { Kommunikationen }\end{array}$} & \multicolumn{2}{|c|}{$\begin{array}{l}\text { Reduzie- } \\
\text { rungsver- } \\
\text { pflichtung }\end{array}$} \\
\hline & Unterz. & Ratif, & Status & Unterz. & Ratif. & Status & 1. & 2. & 3. & 4. & $\mathrm{KP}$ & Real \\
\hline Türkei & & 24.2 .2004 & An.1 & $\begin{array}{ll}-- \\
-\end{array}$ & --- & $-\mathrm{C}_{--}$ & 07 & --- & $-\mathrm{C}_{--}$ & --- & & $0 \%$ \\
\hline Syrien & & 4.1 .96 & & $\begin{array}{ll}-- \\
\end{array}$ & 27.1 .2006 & --- & --- & --- & --- & --- & & \\
\hline Irak & \multicolumn{3}{|c|}{ Keine Vertragspartei } & --- & --- & --- & --- & --- & --- & --- & & \\
\hline Libanon & 12.6.1992 & 15.12.1994 & & --- & 13.11.2006 & --- & $\mathrm{x}$ & --- & --- & --- & & \\
\hline Jordanien & 11.6 .1992 & 12.11.1993 & & --- & 17.3 .2003 & --- & $\mathrm{x}$ & --- & --- & --- & & \\
\hline Israel & 4.6 .1992 & 4.6 .1996 & & 16.12 .1998 & 15.3 .2004 & --- & $\mathrm{x}$ & --- & --- & --- & & \\
\hline Palästina & \multicolumn{3}{|c|}{ Keine Vertragspartei } & --- & --- & --- & --- & --- & --- & --- & & \\
\hline Deutschland & 1.6.1992 & 9.12.1993 & An. 1 & 29.4.1998 & 31.5 .2002 & An.B & $\mathrm{x}$ & $\mathrm{x}$ & $\mathrm{x}$ & $\mathrm{X}$ & $-8 \%$ & $-21 \%$ \\
\hline
\end{tabular}

Quelle: <http://unfccc.int/parties_and_observers/items/2704.php >

b) den Konflikt über die Nutzung der Wasserressourcen des Euphrat und des Tigris zwischen der Türkei, Syrien und dem Irak sowie über die kurdische Minderheit in diesen Staaten und im Iran;

und c) den Irakkonflikt (2003).

Als Folge dieser Konfliktmuster überwiegt im Sicherheitsdiskurs in der Türkei, in Israel und den Maschrekstaaten ein militärisch geprägter Begriff nationaler Sicherheit, während Konzepte der Umweltsicherheit und der menschlichen Sicherheit - außer in Jordanien - keine wesentliche Rolle spielen. ${ }^{9}$

Das Problembewusstsein zu Fragen des Klimawandels ist in den politischen Eliten der Staaten, die über Jahrtausende Opfer der natürlichen Variabilität des Klimas waren, ${ }^{10}$ gering. Die Türkei ist der UN-Rahmenkonvention zum Klimawandel erst am 24.5.2004 beigetreten (Tabelle 1), am 5. Februar 2009 ratifizierte die Türkei das Kyoto-Protokoll und legte dem UNFCCC Sekretariat 2007 die erste nationale Kommunikation vor. Der Irak ist keiner Klimavereinbarung beigetreten. Jordanien ratifizierte das Kyoto-Protokoll im März 2003, Israel folgte im März 2004, Syrien und Libanon im Januar und November 2006. Nur Syrien legte bisher keine nationale Kommunikation vor.

\subsection{Doppelte Herausforderung: Bevölkerungs- wachstum und Klimawandel}

Da der Klima- und Umweltwandel weitgehend anthropogen verursacht wurde, kommt der Bevölkerungsentwicklung und

9 Vgl. M. Aydin: "Security Conceptualisation in Turkey", in: H.G. Brauch et al. (Hrsg.): Security and Environment in the Mediterranean. Conceptualising Security and Environmental Conflicts (Berlin-Heidelberg: Springer 2003): 345356; E. Kam: "Conceptualising security in Israel", in: H.G. Brauch et al., a.a.O.: 357-366; in: H.G. Brauch et al., a.a.O.: 333-344, H.G. Brauch: "Environment and Security in the Middle East: Conceptualizing Environmental, Human, Water, Food, Health and Gender Security", in: C. Lipchin, E. Pellant, D. Saranga, A. Amster, Allyson (Hrsg.): Integrated Water Resources Management and Security in the Middle East (Dordrecht: Springer, 2007): 121-161.

10 Vgl. A.S. Issar, M. Zohar: Climate Change - Environment and Civilization in the Middle East (Berlin u.a.: Springer 2004), dies.: "Climate Change Impacts on the Environment and Civilization in the Near East", in: H.G. Brauch u.a. (2009): 119-130.. der Nachfrage nach Wasser, landwirtschaftlich nutzbaren Böden und Nahrungsmitteln große Bedeutung zu. Die gesamte Region weist (1950-2005) ein starkes Bevölkerungswachstum (Tabelle 2) und eine hohe Urbanisierungsrate (Tabelle 3) auf.

Die Bevölkerung dieser Region ist von 1950 bis 2005 nach VNAngaben von 34,538 Mio. auf 139,869 Mio. angewachsen und nach mittleren Schätzungen soll die Bevölkerung bis 2050 um weitere 92,446 Mio, steigen. Ein beträchtlicher Teil des Bevölkerungszuwachses erfolgte in Städten, teilweise als Folge der Landflucht und der Veränderung der Beschäftigungsstruktur.

Als Folge der Bevölkerungszunahme und der wachsenden Urbanisierung wuchs der Energieverbrauch und stiegen die anthropogenen Treibhausgasemissionen an. Durch zunehmende Wasserknappheit in dieser semiariden und ariden Zone und durch den Verbrauch landwirtschaftlicher Flächen durch die Urbanisierung sank (Ausnahme: Türkei) die Selbstversorgungsrate mit Getreide und stieg der Bedarf an Importen in den meisten Ländern an.

\subsection{Klimawandel und Naturkatastrophen im Nahen Osten}

Die Arbeitsgruppe II des IPCC gelangte $2007 \mathrm{zu}$ folgenden Ergebnissen für Westasien ${ }^{11}$ :

- Der Temperaturanstieg betrug im 20. Jahrhundert in Teilen Asiens zwischen $1^{\circ}$ und $3^{\circ} \mathrm{C}$ (S. 472). Es wird angenommen, dass die Erwärmung im 21. Jahrhundert im Binnenland Westasiens am größten sein wird (S. 478). Nach Projektionen der Klimamodelle sollen die Temperaturen bis 2020 zwischen $1,06^{\circ}$ und $1,55^{\circ} \mathrm{C}$, bis 2050 zwischen $2,0^{\circ}$ und $3,7^{\circ} \mathrm{C}$ und bis 2080 zwischen $2,7^{\circ}$ und $6,3^{\circ} \mathrm{C}$ steigen.

- Der IPCC nimmt an, dass der Meeresspiegel in Asien um 3,1 $\mathrm{mm} /$ Jahr steigen wird (S. 479).

11 IPCC: Climate Change 2007. Impacts, Adaptation and Vulnerability. Working Group II Contribution to the Fourth Assessment Report of the IPCC (Cambridge - New York: Cambridge University Press, 2007). 
Tabelle 2: Bevölkerungswachstum im Nahen Osten (1850-2050).

\begin{tabular}{|c|c|c|c|c|c|c|c|c|c|}
\hline \multicolumn{6}{|c|}{ Reale Bevölkerungsentwicklung } & \multicolumn{2}{|c|}{$\begin{array}{l}\text { Projektion } \\
\text { Mittl. Var. }\end{array}$} & \multicolumn{2}{|c|}{ Veränderungen } \\
\hline & 1850 & 1900 & 1950 & 1980 & 2005 & 2025 & 2050 & $\begin{array}{l}1950- \\
2050\end{array}$ & $\begin{array}{r}2005- \\
2050\end{array}$ \\
\hline 1) Türkei & 10,0 & 13,0 & 21,484 & 46,316 & 72,970 & 89,957 & 98,946 & 77,462 & 25,976 \\
\hline 2) Syrien & 1,5 & 1,75 & 3,536 & 8,971 & 18,894 & 27,519 & 34,887 & 31,351 & 15,993 \\
\hline 3) Irak & 1,25 & 2,25 & 5,340 & 14,093 & 27,996 & 43,293 & 61,942 & 56,602 & 33,946 \\
\hline 4) Libanon & 0,35 & 0,5 & 1,443 & 2,785 & 4,011 & 4,784 & 5,221 & 3,778 & 1,210 \\
\hline 5) Jordanien & 0,25 & 0,3 & 0,472 & 2,225 & 5,544 & 8,029 & 10,527 & 10,055 & 4,983 \\
\hline 6) Israel & & & 1,258 & 3,764 & 6,692 & 8,722 & 10,527 & 9,269 & 3,835 \\
\hline 7) Palästina & 0,35 & 0,5 & 1,005 & 1,476 & 3,762 & 6,553 & 10,265 & 9,260 & 6,503 \\
\hline Summe (1-7) & 13,7 & 18,3 & 34,538 & 79,63 & 139,869 & 188,857 & 232,315 & 197,777 & 92,446 \\
\hline
\end{tabular}

Quelle: Population Division of the Department of Economic and Social Affairs of the United Nations Secretariat. World Population Prospects: The 2006 Revision and World Urbanization Prospects: The 2005 Revision, <http://esa.un.org/unpp> (4.2.2009).

Tabelle 3: Veränderung der Urbanisierungsrate (1950-2030).

\begin{tabular}{|c|c|c|c|c|c|c|c|c|c|c|c|}
\hline & 1950 & 1960 & 1970 & 1980 & 1990 & 2005 & 2010 & 2020 & 2030 & 2040 & 2050 \\
\hline 1) Türkei & 24,8 & 31,5 & 38,2 & 43.8 & 59,2 & 67,3 & 69,6 & 74.0 & 77,7 & 81,1 & 84,0 \\
\hline 2) Syrien & 30,6 & 36,8 & 43,3 & 46,7 & 48,9 & 53,2 & 55,9 & 59,0 & 64,0 & 69,2 & 73,9 \\
\hline 3) Irak & 35,1 & 42,9 & 56,2 & 65,5 & 69,7 & 66,9 & 66,4 & 67,3 & 70,5 & 73,3 & 77,8 \\
\hline 4) Libanon & 32,0 & 42,3 & 59,5 & 73,7 & 83,1 & 86,6 & 87,2 & 88,6 & 90,00 & 91,3 & 92,4 \\
\hline 5) Jordanien & 37,0 & 50,9 & 56,0 & 59,9 & 72,2 & 78,3 & 78,5 & 79,6 & 82,0 & 84,3 & 86,4 \\
\hline 6) Israel & 71,0 & 76,8 & 84,2 & 88,6 & 90,4 & 91,6 & 91,7 & 92,2 & 93,0 & 93,9 & 94,6 \\
\hline 7) Palästina & 37,7 & 44,0 & 54,3 & 62,4 & 67,9 & 71,6 & 72,1 & 74,1 & 77,2 & 80,3 & 83,0 \\
\hline Westasien & 28,6 & 36,0 & 44,6 & 51,9 & 61,0 & 65,0 & 66,3 & 69,1 & 72,5 & 76,0 & 79,2 \\
\hline
\end{tabular}

Quelle: Population Division of the Department of Economic and Social Affairs of the United Nations Secretariat, World Population Prospects: The 2006 Revision and World Urbanization Prospects: The 2007 Revision; bei: <http://esa.un.org/unup> (4.2.2009).

- Teilweise konnte im 20. Jahrhundert ein Rückgang der Niederschläge beobachtet werden. Nach den Projektionen der Klimamodelle sollen die Niederschläge bis 2020 in Teilen des Iran um bis zu 18\% steigen, in anderen Teilen des Nahen Ostens um bis zu 8\% sinken; bis 2050 schwanken die Projektionen zwischen einem Anstieg um 29\% und einer Abnahme um 9\%; sowie bis 2080 zwischen einem Anstieg um 52\% und einer Abnahme um 25\%.

- Als Folge des Klimawandels stieg die Zahl und Intensität von Dürreperioden im Nahen Osten an.

Die Region Westasien ist in Bezug auf Wasserressourcen, Landwirtschaft und Landdegradierung höchst verwundbar, hinsichtlich der Biodiversität, Küstenökosysteme und Siedlungen jedoch kaum (S. 497). Angaben zu Klimafolgen in Westasien sind dürftig bzw. fehlen.

Die vier angeführten Trends werden sich vor allem auf den Wasser- und Agrarsektor in dem semiariden und ariden Nahen Osten negativ auswirken.

\subsection{Umweltstress und gesellschaftliche Folgen des Klimawandels}

Der WBGU-Bericht zum Sicherheitsrisiko Klimawandel unterschied zwischen vier klimarelevanten Konfliktkonstellationen, worunter der WBGU „typische Wirkungszusammenhänge an der Schnittstelle zwischen Umwelt und Gesellschaft [versteht], deren Dynamik zu gesellschaftlicher Destabilisierung oder Gewalt führen kann.“

- Konfliktkonstellation „Klimabedingte Degradation von Süßwasserressourcen": Die Wasserknappheit kann durch die wachsende Nachfrage zu Verteilungskonflikten führen. Die Mechanismen zur Konfliktlösung sind häufig überfordert, was in Destabilisierung und Gewalt resultieren kann.

- Konfliktkonstellation „Klimabedingter Rückgang der Nahrungsmittelproduktion“: Der Rückgang kann zu regionalen Ernährungskrisen führen, wodurch gewalttätige Konflikte begünstigt werden.

- Konfliktkonstellation „Klimabedingte Zunahme von Sturm- und Flutkatastrophen“: Für den Nahen Osten sind anhaltende 
Abb.2: Konfliktkonstellationen in ausgewählten Brennpunkten. Quelle: WBGU (2007: 4).

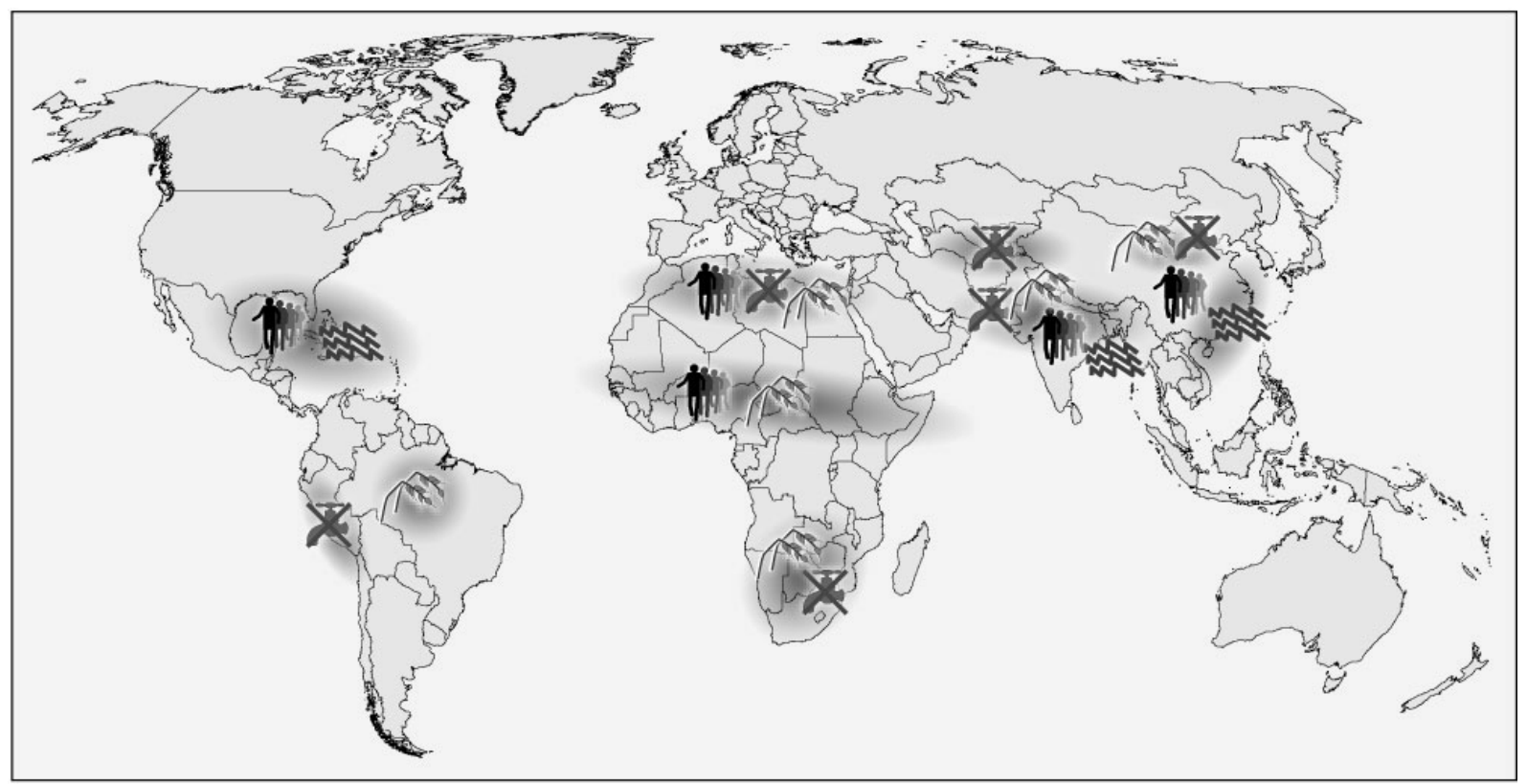

Konfliktkonstellationen in ausgewählten Brennpunkten

Dürreperioden und Hitzewellen die zentrale Herausforderung.

- Konfliktkonstellation „Umweltbedingte Migration“: Migration kann die Konfliktwahrscheinlichkeit in Transit- und Zielregionen erhöhen. Die Zunahme von Dürren, Bodendegradation und Wasserknappheit in Verbindung mit hohem Bevölkerungswachstum, instabilen Institutionen und Armut birgt hohe Risiken für die Entstehung und Verstärkung von innerstaatlicher und internationaler Umweltmigration.

Der WBGU-Bericht hat die möglichen sicherheitspolitischen Folgen für Südeuropa und Nordafrika ${ }^{12}$ - nicht jedoch für den Nahen Osten ${ }^{13}$ - erörtert (Abb. 3).

Das EU-Papier zu „Klimawandel und internationaler Sicherheit" vom 14. März 2008 widmet unter den sechs regionalen Schwerpunkten dem Nahen und Mittleren Osten besondere Aufmerksamkeit, da sich hier „der Migrationsdruck ... sowie die politische Instabilität und die Konflikte ... in Zukunft verstärken" können.

12 Vgl. H.G. Brauch: „Destabilisierungs- und Konfliktpotential prognostizier ter Umweltveränderungen in der Region Südeuropa und Nordafrika bis 2020/2050“, bei: <http://www.wbgu.de/ wbgu_jg2007_ex01.pdf>.

13 Vgl. H.G. Brauch: "Impact of Global Warming and Non-Conventional Water Resources: Potential of solar thermal desalination to defuse water as a conflict issue - A conceptual contribution to conflict resolution and long-term conflict avoidance", in: H. Shuval; H. Dweik (Hrsg.): Israel-Palestinian Water Issues - from Conflict to Cooperation (Berlin - Heidelberg: Springe, 2007): 357 378; L. Freimuth; G. Bromberg, M. Mehyar; N. Al Kahteeb: Climate Change: $A$ New Threat to Middle East Security (Amman - Bethlehem - Tel-Aviv: Eco Peace - Friends of the Earth Middle East, 2007); at: < http://www.foeme.org/index images/dinamicas/publications/publ78_1.pdf $>$.
Im Bericht zur Implementierung der Europäischen Sicherheitsstrategie hat der Europäische Rat ausdrücklich den Klimawandel als Sicherheitsfrage und den Nahen und Mittleren Osten als zentrale Region ihrer Sicherheitspolitik genannt. ${ }^{14}$ Alle vier Konfliktkonstellationen des WBGU sind für diese Region relevant.

\subsection{Konfliktkonstellation „Klimabedingte De- gradation von Süßwasserressourcen“}

Es bestehen folgende Konflikte über Zugang, Kontrolle und Verteilung von Wasserressourcen, die zu politischen Spannungen und Konflikten, aber zu keinem Wasserkrieg geführt haben:

- Das türkische GAP Projekt, durch das Wasser des Euphrat und Tigris durch zahlreiche Dämme intensiv landwirtschaftlich genutzt wird, hat zu Konflikten mit Syrien und Irak geführt; ${ }^{15}$

14 Report on the Implementation of the European Security Strategy - Providing Security in a Changing World - (submitted to the European Council of 11-12 December 2008), Brussels 11 December 2008.

15 Vgl. W. Scheumann: "The Euphrates Issue in Turkish-Syrian Relations", in: H.G. Brauch u.a. (2003):745-760; M. Aydin, F. Ereker: "Water Scarcity and Political Wrangling: Security in the Euphrates and Tigris Basin", in: H.G. Brauch u.a. (2009): 603-614. 
- Konflikt zwischen Israel und seinen Nachbarn über das Wasser des Jordans; ${ }^{16}$

- Konflikt zwischen Israel und Syrien über die Kontrolle der Wasserressourcen der besetzten Golanhöhen und zwischen Israel und dem Libanon über den Litany Fluss;

- die ungelöste Frage des Zugangs zur Nutzung der gemeinsamen Grundwasserreservoirs durch Israel und die palästinensische Autonomiebehörde. ${ }^{17}$

Die Wasserfrage erhöht die Dringlichkeit der Lösung des Nahostkonflikts. Die Probleme des Trinkwassers lassen sich am ehesten mit einer multilateralen Kooperationsstrategie und durch neue Technologien lösen.

\subsection{Konfliktkonstellation „Klimabedingter Rück- gang der Nahrungsmittelproduktion“}

Im Nahen und Mittleren Osten ist der Grad der Selbstversorgung mit Getreide seit den 1950er Jahren durch das hohe Bevölkerungswachstum, die Zunahme des Fleischverzehrs und durch Klimaveränderungen gesunken. Nach Projektionen der FAO wird der Importbedarf an Getreideprodukten bis 2030 dramatisch steigen. ${ }^{18}$ Steigende Lebensmittelpreise haben bereits wiederholt zu Massenprotesten und Brotunruhen geführt. Die für die wachsende Bevölkerung des Nahen Ostens benötigten Lebensmittel lassen sich mit herkömmlichen Mitteln in den semiariden und ariden Gebieten nicht erzeugen. Neue Methoden der wirtschaftlichen Nutzung der Trockengebiete (Modell von Almeria; Erzeugung von Produkten mit hohem finanziellen Ertrag) können die Boden- und die Nahrungssicherheit aber durchaus erhöhen. ${ }^{19}$

\subsection{Konfliktkonstellation „Klimabedingte Zu- nahme von Sturm- und Flutkatastrophen“}

Diese Konfliktkonstellation trifft für den Nahen Osten nicht zu. Dagegen haben hier Dürreperioden in den letzten Jahrtausenden wiederholt zum Zusammenbruch von Hochkulturen geführt und zur Massenmigration beigetragen. Da mit dem Klimawandel auch Dürreperioden und Hitzewellen zunehmen werden, ist diese Region ganz besonders gefährdet.

16 Vgl. I. Dombrowsky: "Water Accords in the Middle East Peace Process: Moving towards Cooperation”, in: H.G. Brauch u.a. (2003): 729-744; Shuvall, Dweik (2007), a.a.O.

17 Vgl. Shuval, Dweik (2007); B. Hayek: "Water Resources in the Arab World: A Case Study on Jordan"; J. Selby: "New Security Thinking' in Israeli-Palestinian Water Relations"; A. Jägerskog: "Functional Water Cooperation in the Jordan River Basin: Spillover or Spillback for Political Security? “; alle in: H.G. Brauch u.a. (2009); A. Issar: "Progressive Development of the Water Resources of Israel and Palestine to Mitigate the Negative Impact of Global Warming; H.S. Salem: "Social and Environmental Impacts of Climate Change for the Eastern Mediterranean", alle in: H.G. Brauch u.a. (2010).

18 FAO: World agriculture: towards 2015/2030. An FAO Perspective (Rome: FAO; London: Earthscan, 2003).

19 Vgl. A.M. García Lorca: "Agriculture in Drylands: Experience in Almería"; U. Safriel: "Alternative Liveli hoods for Attaining Sustainability and Security in Drylands"; beide in: Brauch u.a. (2010).

\subsection{Konfliktkonstellation „Umweltbedingte Mig- ration"}

Zahlreiche Gründe haben zur Migration im Nahen Osten beigetragen. Mit Gründung des Staates Israel (1948) und nach dem Zerfall der Sowjetunion (1991) sind vor allem in den 1950er und 1990er Jahren mehr Menschen in die Region eingewandert, während im Zeitraum von 1960-1990 über 2,2 Millionen Menschen die Region verlassen haben (vor allem aus der Türkei). Der Irak war bis zu den Kriegen von 1991 und 2003 ein Land, das über eine Million Arbeitsmigranten aus anderen arabischen Ländern anzog, aber seit 2003 sind die meisten Migranten zurückgekehrt und Millionen Iraker sind nach Jordanien und Syrien geflohen.

Das EU-Papier zu ,Klimawandel und internationale Sicherheit' hat die umweltbedingte Migration als eine von sieben zentralen Bedrohungen aufgeführt und hierzu festgestellt:

Die Lage derjenigen Teile der Bevölkerung, die bereits unter schlechten Gesundheitsbedingungen, Arbeitslosigkeit oder sozialer Ausgrenzung leiden, wird durch die Folgen des Klimawandels noch prekärer, was Migrationsbewegungen innerhalb eines Landes oder zwischen Ländern auslösen bzw. noch verstärken könnte. ... Einige Länder, die durch den Klimawandel extrem gefährdet sind, fordern bereits eine internationale Anerkennung der umweltbedingten Migration. Eine solche Migration kann zunehmend zu Konflikten in den Transit- und Zielgebieten führen. Europa muss auf einen wesentlich erhöhten Migrationsdruck gefasst sein.

Neben Schätzungen von Wissenschaftlern ${ }^{20}$, die von den Vereinten Nationen, der EU und auch der CIA zitiert werden, gibt es weltweit und für diese Region keine systematischen Statistiken (UNPP, UNHCR, UNPF, OECD), da Gründe für die Binnenmigration (Landflucht, Urbanisierung) in den Zensusdaten nicht erfasst werden und Umweltaspekte keinen Rechtsgrund für die Gewährung von Asyl schaffen. Die wenigen empirischen Fallstudien sind weder repräsentativ noch vergleichbar. Systematische wissenschaftliche Analysen zur relativen Bedeutung der durch Umweltfaktoren im Nahen Osten ausgelösten Migration fehlen, was jedoch nicht den Schluss zulässt, dass Umweltfaktoren als Migrationsgrund irrelevant sind. ${ }^{21}$

\section{Klimafolgen als Sicherheitsgefährdung}

Von den vier Konfliktkonstellationen des WBGU gefährden die Verknappung von Süßwasserressourcen und die steigende Importabhängigkeit im Bereich der Lebensmittel in Verbindung mit anziehenden Preisen die Verwundbarkeit. Dürreperioden stellen eine Gefahr für die menschliche Sicherheit dar und

20 Vgl. N. Myers: Environmental Exodus. An Emergent Crisis in the Global Arena (Washington, DC: Climate Institute, 1995); ders.: "Environmental Refugees: A growing Phenomenon of the 21st Century", in: The Philosophical Transactions of the Royal Society (Biological Sciences), April 2002.

21 Im Rahmen des EU-Forschungsprojekts zur Umweltmigration (EACHFOR) behandelt nur die Arbeit von Z. Kadirbeyoglu zur Türkei ein Land des Nahen Ostens. Sie stellt das Fehlen von Umweltfaktoren in der Migrationsliteratur zur Türkei fest. Skeptisch sind: C. Raleigh, L. Jordan, I. Salehyan: Assessing the Impact of Climate Change on Migration and Conflict, paper for the 'Social Dimensions of Climate Change' workshop, World Bank, Washington, D.C. 5-6 March 2008, bei: <http://siteresources.world-bank.org/ EXTSOCIALDEVELOPMENT/Resources/SDCCWorkingPaper_MigrationandConflict.pdf > . 
konfrontieren vor allem die Armen mit einem Überlebensdilemma: Sie stehen vor der Wahl, ihre Heimat zu verlassen und in die (Slums der) Städte zu ziehen, um dort eine Überlebensperspektive zu suchen, oder aber zurückzubleiben und die verschlechterten Lebensbedingungen zu ertragen. Die wachsende menschliche Unsicherheit verursacht nicht zwingend nationale oder internationale Sicherheitsprobleme und nur in Ausnahmefällen führen diese zu gewaltsamen Konflikten um knappes Wasser, fruchtbare Böden und Nahrung. ${ }^{22}$ In dieser konfliktintensiven Region ist der Klimawandel nie der Hauptgrund, allenfalls ein Auslöser für ein erneutes Ausbrechen bestehender und ungelöster historischer Konflikte.

\section{Politische Antworten: Transformations- strategien}

Der deutsch-britische und der EU-Ansatz zur Behandlung der Wechselbeziehungen zwischen Klimawandel und Sicherheit ist präventiver Natur. ${ }^{23}$ Präventive sicherheitspolitische Überlegungen erfordern komplexe Transformationsstrategien, die in der Folge den Menschen in den semiariden und ariden, von Wassermangel, Bodenerosion, Desertifikation und Dürre betroffenen Gebieten eine Überlebensperspektive eröffnen und dem Zwang zur umweltbedingten Migration entgegenwirken.

Eine von vielen möglichen Transformationsstrategien berührt das bisher weitgehend ungenutzte technische solare Energiepotenzial in den sonnenreichen Gebieten des Nahen Ostens. Die Nutzung der solarthermischen Stromerzeugung in Wüstengebieten steht an ihrem Anfang und eine, economy of scale' ist im Entstehen. ${ }^{24}$ Die Nutzung von konzentrierten Photovoltaikanlagen kann mittelfristig attraktive Alternativen bieten. Solare Entsalzungsanlagen, die beide Technologien nutzen, können langfristig dazu beitragen, die hohen Kosten für Öl und Gas zu umgehen und sie vermeiden die Proliferationsgefahr, die durch den Transfer von Nukleartechnologien entstehen kann.

Die verstärkte Nutzung alternativer und erneuerbarer Energiequellen in Trockengebieten kann neue Lebensgrundlagen für Millionen Menschen schaffen und damit auch eine ökonomische Grundlage für menschliche, nationale und internationale Sicherheit. Sie kann mittel- und langfristig den Wettbewerb um Zugang und Kontrolle von fossilen Energiequellen entspannen und Staaten, deren Energiereserven zurückgehen, alternative Einkommen gewähren.

22 Diese Zusammenhänge wurden eingehend diskutiert in: Brauch (2002), sowie zum ,Überlebensdilemma', ders.; „From a Security towards a Survival Dilemma", in: ders. u.a. (2008): 537-552.

$23 \mathrm{Vgl}$. den gemeinsamer Beitrag von Bundesaußenminister Frank-Walter Steinmeier und seinem britischen Amtskollegen David Miliband: „Europa muss sich den sicherheitspolitischen Folgen des Klimawandels stellen“, bei: <http://www.auswaertiges-amt.de/diplo/de/Infoservice/Presse/Interviews/2008/080313-klimasicherheit. html $>$.

24 Vgl. D. Faiman: „Solar Energy on a Global Scale: Its Impact on Security”; F. Trieb, W. Krewitt, N. May: "Solar Energy as a Key for Power and Water in the Middle East and North Africa”, in: H.G. Brauch u.a. (2009): 395-426.

\section{Schlussfolgerungen für die Europäische Sicherheit}

Durch einen „Überlebenspakt“ könnte die ökonomische Interdependenz der EU-Staaten mit den Staaten Nordafrikas und des Nahen und Mittleren Ostens (MENA-Region) gefördert werden, wobei das ,virtuelle Wasser', Lebensmittelimporte in die ariden und semiariden Zonen, und die ,virtuelle Sonne', der Export erneuerbarer Energien in die EU-Staaten, verknüpft werden sollten. Ein Überlebenspakt bietet sich für die EU-Staaten und die Staaten der MENA-Region an, da die wechselseitige Abhängigkeit eine Nutzung von Nahrung oder Energie als Waffe verbietet. $^{25}$

Mit dem DESERTEC-Konzept ${ }^{26}$ und der Solarinitiative im Rahmen der Mittelmeerunion/Barcelona-Prozess könnten die EU-Staaten zusammen mit den MENA-Staaten gemeinsam die Grundlage für eine langfristig angelegte und nachhaltige ökonomische Interdependenz schaffen. ${ }^{27}$ Der vereinbarte Mittelmeersolarplan könnte - nach Vorschlägen aus der Wissenschaft - in zwei Phasen zwischen 2008 und 2020 entwickelt werden. ${ }^{28}$

Dieser Mittelmeersolarplan böte den Staaten des Nahen Ostens (außer dem Irak) einen Rahmen für eine langfristige Wirtschaftskooperation, die den Ursachen des Klimawandels entgegenwirken, für die sonnenreichen Staaten den Aufbau neuer Wirtschaftssektoren ermöglichen, in den Trockengebieten Arbeitsplätze und einen Beitrag zu mehr menschlicher und regionaler Sicherheit schaffen und die bei Erreichen einer, economy of scale', Probleme der Wasser-, Boden- und Nahrungssicherheit nachhaltig lösbar machen würde.

25 Vgl. hierzu H.G. Brauch (2002); ders.: "A Survival Pact for the Mediterranean: Linking 'virtual water' and 'virtual sun'”, in: R.K. Pachauri, G. Vasudeva (Hrsg.): Johannesburg and beyond. Towards concrete action. Proceedings of the Colloquium held on 24 March 2002 in New York, U.S.A. (New Delhi: Teri, 2002): 151-190.

26 Vgl. zum DESERTEC Konzept, bei:<http://www.desertec.org/de/concept. html>,

27 Vgl. Am 13.7.2008 haben die Staats- und Regierungschefs der 27 EU-Staaten die EU-Mittelmeerunion angenommen, am 3.und 4. November 2008 haben die Außenminister der 43 Partnerstaaten das Mandat und den institutionellen Rahmen für die Mittelmeerzusammenarbeit vereinbart. Am 28.-29. Oktober 2008 fand auf Einladung des BMU in Berlin ein Expertenworkshop zum Mittelmeersolarplan statt und am 8. Dezember 2008 folgte ein deutsch-französisches Symposium zu Sonnenenergietechnologien für die Stromerzeugung in der französischen Botschaft in Berlin, an der Vertreter beider Regierungen und der Industrie teilnahmen. Vgl. „German-French Symposium on Solar Energy Technologies for Power Production. PV, solar thermal plants and trans-mediterranean cooperation", Tagung der DENA, 8 December 2008; bei: <http:// www.dena.de/ de/themen/ thema-reg/veranstaltungen/veranstaltung/german-french-symposium/>.

28 Vgl. C. Hoyer-Klick, „The Union for the Mediterranean and the Mediterranean Solar Plan”, bei: <http://www. eurec.be/files/Oldenburg,\%2028th\%20July\%2 02008/07_Union\%20for\%20the\%20Mediterranean.pdf $>$. 